\title{
The cost effectiveness of Single-Photon Emission- Computed Tomography compared with stress echocardiography for diagnosis of coronary artery disease
}

\section{Javad Javan-Noughabi}

Mashhad University of Medical Sciences https://orcid.org/0000-0001-7809-1377

Aziz Rezapour ( $\square$ rezapoor_a57@yahoo.com )

Iran University of Medical Sciences

Marjan Hajahmadi

Iran University of Medical Sciences

Vahid Alipour

Iran University of Medical Sciences

Abdosaleh Jafari

Shiraz University of Medical Sciences

Ahmad Faramarzi

Urmia University of Medical Sciences

Ali Vafaee Najar

Mashhad University of Medical Sciences

\section{Research}

Keywords: Economic Evaluation, Cost Effectiveness Analysis, Coronary Artery Disease, Single-Photon Emission-Computed Tomography, Stress Echocardiography

Posted Date: January 4th, 2021

DOl: https://doi.org/10.21203/rs.3.rs-108261/v2

License: (c) (i) This work is licensed under a Creative Commons Attribution 4.0 International License. Read Full License 


\section{Abstract}

Early diagnosis of coronary artery disease is very vital for success in treatment of this disease. However, the appropriate diagnostic modality for diagnosis of coronary artery disease is uncertain. Therefore, we conducted this study to compare the cost-effectiveness of Single-Photon Emission-Computed Tomography (SPECT) versus stress echocardiography for diagnosis of coronary artery disease in Iran. A decision tree model was developed to compare the cost-effectiveness of SPECT versus stress echocardiography. This study was conducted in Tehran, capital of Iran, between April 1, 2017, and September 1, 2018. The cost analysis was conducted from a societal perspective and medical direct costs, nonmedical direct costs and indirect costs were estimated. Effectiveness was defined as the accurate detection of coronary artery disease and invasive coronary angiography was used as a gold standard. The incremental cost-effectiveness ratio was defined as an additional cost per correct diagnosis. SPECT was associated with higher costs and lower effectiveness compared with stress echocardiography. Therefore, stress echocardiography is dominant alternative compared to SPECT. The results also indicated that total cost per patient were $\$ 970.49$ and $\$ 781.8$ for SPECT and stress echocardiography, respectively. Also, the percent of true positive and true negative responses were $\% 88$ and $\% 79$ for SPECT and $\% 90$ and $\% 92$ for stress echocardiography. The results of this study indicate that stress echocardiography is the cost-effective modality in the diagnosis of coronary artery disease compared with SPECT. According to the results, it is suggested cardiologists use stress echocardiography instead of SPECT for the diagnosis of coronary artery disease. The result of the current study has significant concepts for decision-making in designing clinical guidelines for the diagnosis of coronary artery disease.

\section{Background}

Cardiovascular disease (CVD) has become one of the most common Non-Communicable Diseases (NCDs) throughout the world and it is responsible for about $40 \%$ of all NCD deaths [1-3]. One of the most common CVDs is Coronary artery disease (CAD) that leads to a considerable increase in mortality and morbidity [4]. CAD is responsible for more than $30 \%$ of all deaths and about $40 \%$ of cardiovascular deaths $[4,5]$. Based on previous studies, CAD is accounted for about 500 thousand deaths in the USA and almost 2 million deaths in Europe annually [6]. Also, it is reported that about $50 \%$ of all deaths in Iran are due to CAD [7]. An important point is that about one half of all CAD deaths occurred suddenly and unexpectedly. On the other hand, almost $50 \%$ of CAD deaths in developing countries occurred in people aged below 70 years $[8,9]$. However, CAD is one of the most preventable diseases and it is reported that early diagnosis can lead to about $80 \%$ decrease in deaths relating to CAD $[10,11]$.

Therefore, early diagnosis of CAD is very essential for success in treatment of this disease. Several modalities are used to diagnose CAD including invasive and non-invasive modalities. Invasive strategies such as angiography for CAD diagnosis is expensive and with adverse side effects. Therefore, it is better than cheaper, non-invasive, easily available and more effective modalities are used for diagnosis of CAD [12]. Non-invasive modalities such as stress echocardiography and Single-Photon Emission-Computed 
Tomography (SPECT) diagnose the CADs without break in the skin [13]. Among non-invasive methods, the use of stress echocardiography is widespread in people who are unable to exercise, such as patients with peripheral vascular disease, myocardial infarction, and orthopedic disorders [14]. Stress echocardiography is a very suitable, adaptable and cheap tool with a wide use for non-invasive imaging of the heart [15]. Stress echocardiography works similar to CT-scan, but the advantage of stress echocardiography is the reduction of radiation exposure to the patient compared to CT-scan $[16,15]$. Also, previous studies showed that SPECT has a high diagnostic value in the diagnosis of CAD and has been proposed as an important diagnostic modality for patients [17]. However, there are significant differences between the two diagnostic methods in terms of cost and effectiveness. In various studies, the cost of SPECT is estimated at between $\$ 5,417$ and $\$ 20,550$ per correct diagnosis of heart disease. Also, the cost per QALY for the SPECT method is estimated at $\$ 38,000$ to $\$ 40,316$, which is much higher than the stress echocardiography method $[18,19]$. Although SPECT is much more expensive than stress echocardiography, it has shown better negative predictive value than stress echocardiography $[20,21]$.

Due to significant differences between SPECT and stress echocardiography, economic evaluation studies tried to examine the cost-effectiveness of these diagnostic methods. However, there is no evidence about the superiority of one of these methods [22-24]. Therefore, we conducted this study to evaluate the costeffectiveness of SPECT compared to stress echocardiography in Iran.

\section{Methods}

This cross-sectional, cost-effectiveness study was conducted in three general hospital in Tehran, capital of Iran, between April 1, 2017 and September 1, 2018. We used a decision tree model to find the cost effective modality for diagnosis of CAD. All persons suspected to CAD that used SPECT or stress echocardiography were studied. The response of each diagnostic modality was positive or negative. The prognostic value of this modalities were assessed by invasive coronary angiography as a gold standard [25]. Finally, terminal nodes for each modality in decision tree model were true positive, true negative, false positive, false negative. Decision tree algorithm is shown in Figure 1.

In this study, the societal perspective was selected for calculating the costs. This perspective is including medical direct costs (diagnostic modalities, visits, surgical costs, hoteling, drugs, ECG, laboratory tests, consumables) and nonmedical direct costs (traveling, accommodation, phone) and indirect costs (time costs imposed on patient, time costs imposed on patient's accompany). Medical direct costs were extracted from patient's medical records and non-medical direct and indirect costs were collected by interviewing of the patient. It is necessary to mention that the indirect costs were calculated using human capital approach [26]. In our one-year study period, the discount rate was not used. All costs were converted to US dollars using the average exchange rate Central Bank of Iran for the time period of this study. According to this, one dollar is equal to 36692 Iranian Rials. We used the percent of correct diagnosis (True positive and true negative) as the outcomes of each diagnostic modalities in decision tree model. In this step, invasive coronary angiography used as a gold standard for evaluation of SPECT 
and stress echocardiography in CAD diagnosis [25]. The incremental cost-effectiveness ratio (ICER) was defined as the difference cost to difference effectiveness (correct diagnosis).

We used SPSS 16 and TreeAge 2011 software and a decision tree model for data analysis. TreeAge provides a popular approach for model simplification to compare the cost effectiveness of alternative strategies. Furthermore, we performed a sensitivity analysis for improving the accuracy of the study.

\section{Results}

Table1 shows the demographic characteristics of patients included in this economic evaluation study. A total of 510 patients were included in this study. According to the results of this study, the mean age of patients in SPECT and Stress echocardiography was 60 and 56 years respectively. More than $\% 50$ of patients in both group were woman, married and with university education.

The mean cost of CAD diagnosis in two groups of diagnostic modalities is compared in table 2 . This table shows that medical direct costs and non-medical direct costs in both groups were the highest and lowest cost respectively. As seen in table 2, the surgical costs were the highest type of medical direct costs in both group (233.75 and 201.8 US\$ in SPECT and Stress Echocardiography, respectively). The highest non-medical direct cost was related to traveling (\$49.7 in SPECT group vs \$43 in Stress echocardiography group). The time costs imposed on patients were the highest type of indirect costs in both arms (\$88.7and \$76.3 in SPECT and Stress echocardiography, respectively).

Cost-Effectiveness results:

The results of cost-effectiveness analysis of SPECT compared with stress echocardiography have been indicated in Table 3. The results showed that Stress echocardiography have an expected cost equal to $\$ 316.3$ s and an expected effectiveness equal to 0.84 . Our results also showed that in the SPECT arm, the expected cost was $\$ 656.6$ dollars and the expected effectiveness was 0.69 . As a result, stress echocardiography is dominant compared to SPECT (less costly and more effective).

Cost effectiveness results of two diagnostic modalities were compared in figure 2. The dominant strategy, Stress echocardiography, is shown with a square in figure 2 (less costly and more effective). Also, SPECT as a dominated strategy is shown with the triangle (more costly and less effective).

Sensitivity analysis

To cope with uncertainty, we performed a sensitivity analysis that shown in Tornado diagram (Figure 3). In one-way sensitivity analysis, we increased all parameters to $20 \%$. Then, we examined the changes in the ICER using one-way sensitivity analysis. Tornado diagram showed that ICER has the highest sensitivity to changing in the true positive cases in the stress echocardiography arm.

\section{Discussion}


The purpose of any economic evaluation study, especially in the field of medical decision-making, is to raise the awareness of policy makers about the costs and effectiveness of alternative therapies, technologies and interventions for a disease. Therefore, we conducted this study to evaluate the costeffectiveness of SPECT compared to stress echocardiography in Iran.

According to the results of our study, the mean of medical direct costs was the highest cost in both groups. Several economic evaluation showed that medical direct costs constitute the largest portion of costs among patients with CAD and other disease such as cancer [27-29]. The results of medical direct costs for diagnostic modalities (SPECT and stress echocardiography) showed that SPECT was more expensive than stress echocardiography (\$233.3 vs $\$ 131.8$ ). Also, total medical direct costs per patient (the costs of diagnostic modalities plus angiography) were $\$ 740.6$ in the branch of SPECT and \$583 in the branch of stress echocardiography. Both of the non-medical direct costs and indirect costs in the branch of SPECT was more than stress echocardiography. Lee et al in a study entitled "Cost-effectiveness of coronary CT angiography in patients with chest pain: Comparison with myocardial single photon emission tomography" showed that the medical direct cost for SPECT is \$332. In this study, the cost of angiography was estimated about $\$ 601$ [30]. In another study, the cost of SPECT cost was $\$ 300$ whereas the cost of stress echocardiography was $\$ 120$, and for coronary angiography was $\$ 1200$ [31]. Hlatky et al in an economic outcomes study calculated the costs of Coronary Anatomy Imaging modalities. In this study, the costs of SPECT, PET and computed tomography angiography (CTA) were calculated for 2years. The most expensive and cheapest diagnostic modalities were PET and SPECT respectively. The mean costs of PET, CTA and SPECT for 2-years were $\$ 6,647, \$ 4,909$ and $\$ 3,965$ [32]. Van der Wall et al. conducted a cost analysis study on diagnostic modalities (including CTA, PET and SPECT) for diagnosis of suspected CAD. This study showed that SPECT is much more cheaper than CTA and PET over 2 years follow-up of suspected CAD patients [33]. However, it is reported that SPECT is significantly more expensive compared with stress echocardiography for CAD diagnosis [34].

In this cost-effectiveness study, we estimated the percent of true positive and true negative responses for each diagnostic modality. The percent of true positive and true negative responses were $\% 88$ and $\% 79$ for SPECT and $\% 90$ and $\% 92$ for stress echocardiography. The results of previous studies about comparison of correct responses of this two diagnostic modalities were Contradictory. However, it is claimed that stress echocardiography is a useful diagnostic technology for CAD diagnosis. In an evidence-based study, the overall pooled sensitivity and specificity for stress echocardiography was 0.80 and 0.84 using invasive angiography as a gold standard [35]. A systematic review analysis showed that sensitivity for stress echocardiography can be variable between $\% 53$ and \%91 and specificity between $\% 44$ and \%94 [36]. sensitivity and specificity for SPECT reported $\% 70$ and $\% 76$ respectively [37]. However, another study showed that sensitivity and specificity of SPECT in CAD diagnosis was $\% 83$ and $\% 89$ [38].

The results of this study revealed that stress echocardiography has lower costs and more effectiveness (dominant) compared with SPEC and stress echocardiography is the most cost-effective strategy against SPECT. Many studies evaluated the cost effectiveness of non-invasive Imaging modalities for the diagnosis of CAD. Javan-Noughabi et al in a systematic review on economic evaluation of SPECT for 
diagnosis of CAD indicated that SPECT is an attractive strategy compared with PET and CTA. But, SPECT in comparison with stress echocardiography, DECT and CMR was a dominated modality [39]. In a systematic review on the cost effectiveness of stress echocardiography against other non-invasive imaging modalities for the diagnosis of CAD, the results showed that stress echocardiography is cost effective compared with SPECT in the majority of included study [35]. Bedetti et al in a study entitled "Economic analysis including long-term risks and costs of alternative diagnostic strategies to evaluate patients with chest pain" showed that the cost of SPECT is more than 2 times expensive than stress echocardiography. In this study, stress echocardiography was the dominant modality versus SPECT (ICER defined as cost per patient correctly identified) [40]. One obvious limitation in this economic evaluation study was that decision tree model used for short treatment period (1-year), whereas CAD is a chronic disease requiring long-term treatment. This may limit the generalizability of results.

\section{Conclusions}

The results indicate that stress echocardiography is the most cost-effective strategy against SPECT in diagnosis of coronary artery disease. According to the results, it is suggested cardiologists use stress echocardiography instead of SPECT for the diagnosis of coronary artery disease. The result of the current study has significant concepts for decision-making in designing clinical guidelines for the diagnosis of coronary artery disease.

\section{Declarations}

\section{Funding:}

No funding was received for this research.

\section{Competing interests:}

The authors declare that they have no Competing interests.

\section{Availability of data and materials:}

All data are available on reasonable request.

\section{Authors' contributions:}

Designing the study, data collection, analysis, drafting the first version of the article and finalization of the article conducted by JJN. AR, MH and VA cooperated in study design and data collection. Other authors provided critical comments on this manuscript. All authors read and approved the final manuscript.

\section{Ethics approval and consent to participate:}


Ethical approval for this study was obtained from Ethics Committee of the Iran University of Medical Sciences. Informed and verbal consent was obtained from participants before data collection.

\section{Consent for publication:}

Consent for publication is not applicable as this study did not include names, images, or videos relating to individual participants.

\section{Acknowledgements}

The present article was extracted from the PhD thesis written by Javad Javan-Noughabi and was supported by Iran University of Medical Sciences grants No. IUMS/SHMIS_9321504006. The authors would like to thank the Research Vice-Chancellor of Iran University of Medical Sciences for supporting the research. We also acknowledge the participants who helped us do this research with their support and cooperation.

\section{References}

1. Sanchis-Gomar, F., Perez-Quilis, C., Leischik, R., \& Lucia, A. (2016). Epidemiology of coronary heart disease and acute coronary syndrome. Annals of translational medicine, 4(13).

2. Benjamin, E. J., Virani, S. S., Callaway, C. W., Chamberlain, A. M., Chang, A. R., Cheng, S., et al. (2018). Heart disease and stroke statistics-2018 update: a report from the American Heart Association. Circulation.

3. Virani, S. S., Alonso, A., Benjamin, E. J., Bittencourt, M. S., Callaway, C. W., Carson, A. P., et al. (2020). Heart disease and stroke statistics-2020 update: a report from the American Heart Association. Circulation, E139-E596.

4. Hanson, M. A., Fareed, M. T., Argenio, S. L., Agunwamba, A. O., \& Hanson, T. R. (2013). Coronary artery disease. Primary Care: Clinics in Office Practice, 4O(1), 1-16.

5. Gao, R., Yang, Y., Han, Y., Huo, Y., Chen, J., Yu, B., et al. (2015). Bioresorbable vascular scaffolds versus metallic stents in patients with coronary artery disease: ABSORB China trial. Journal of the American College of Cardiology, 66(21), 2298-2309.

6. Wolber, T., Maeder, M., Rickli, H., Riesen, W., Binggeli, C., Duru, F., et al. (2007). N-terminal pro-brain natriuretic peptide used for the prediction of coronary artery stenosis. European journal of clinical investigation, 37(1), 18-25.

7. Hatmi, Z., Tahvildari, S., Motlag, A. G., \& Kashani, A. S. (2007). Prevalence of coronary artery disease risk factors in Iran: a population based survey. BMC cardiovascular disorders, 7(1), 32.

8. Sudzinova, A., Stancak, B., Sedlak, J., Misikova, S., Olexa, P., \& Spurny, P. (2009). The effect of myocardial revascularization on malignant ventricular arrhythmias in coronary artery disease. Bratis/ Lek Listy, 110(4), 226-232. 
9. Nag, T., \& Ghosh, A. (2013). Cardiovascular disease risk factors in Asian Indian population: A systematic review. Journal of cardiovascular disease research, 4(4), 222-228.

10. Alizadehsani, R., Habibi, J., Bahadorian, B., Mashayekhi, H., Ghandeharioun, A., Boghrati, R., et al. (2012). Diagnosis of coronary arteries stenosis using data mining. Journal of medical signals and sensors, 2(3), 153.

11. Karimi-Moonaghi, H., Mojalli, M., \& Khosravan, S. (2014). Psychosocial complications of coronary artery disease. Iranian Red Crescent Medical Journal, 16(6).

12. Joloudari, J. H., Hassannataj Joloudari, E., Saadatfar, H., GhasemiGol, M., Razavi, S. M., Mosavi, A., et al. (2020). Coronary artery disease diagnosis; ranking the significant features using a random trees model. International journal of environmental research and public health, 17(3), 731.

13. Schächinger, V., Britten, M. B., \& Zeiher, A. M. (2000). Prognostic impact of coronary vasodilator dysfunction on adverse long-term outcome of coronary heart disease. Circulation, 101(16), 18991906.

14. Sicari, R., Nihoyannopoulos, P., Evangelista, A., Kasprzak, J., Lancellotti, P., Poldermans, D., et al. (2008). Stress echocardiography expert consensus statement: European Association of Echocardiography (EAE)(a registered branch of the ESC). European Journal of Echocardiography, 9(4), 415-437.

15. Marwick, T. H. (2018). Stress echocardiography. In Echocardiography (pp. 491-519): Springer.

16. Varga, A., Garcia, M. A. R., Picano, E., \& Registry, I. S. E. C. (2006). Safety of stress echocardiography (from the international stress echo complication registry). The American journal of cardiology, 98(4), 541-543.

17. Hachamovitch, R., Berman, D. S., Kiat, H., Cohen, I., Cabico, J. A., Friedman, J., et al. (1996). Exercise myocardial perfusion SPECT in patients without known coronary artery disease: incremental prognostic value and use in risk stratification. Circulation, 93(5), 905-914.

18. Marcassa, C., Bax, J. J., Bengel, F., Hesse, B., Petersen, C. L., Reyes, E., et al. (2008). Clinical value, cost-effectiveness, and safety of myocardial perfusion scintigraphy: a position statement. European heart journal, 29(4), 557-563.

19. Garber, A. M., \& Solomon, N. A. (1999). Cost-effectiveness of alternative test strategies for the diagnosis of coronary artery disease. Annals of internal medicine, 130(9), 719-728.

20. Hashimoto, A., Nakata, T., Wakabayashi, T., Kusuoka, H., \& Nishimura, T. (2009). Incremental prognostic value of stress/rest gated perfusion SPECT in patients with coronary artery disease. Circulation Journal, 73(12), 2288-2293.

21. van Werkhoven, J. M., Schuijf, J. D., Gaemperli, O., Jukema, J. W., Boersma, E., Wijns, W., et al. (2009). Prognostic value of multislice computed tomography and gated single-photon emission computed tomography in patients with suspected coronary artery disease. Journal of the American College of Cardiology, 53(7), 623-632.

22. Dragomir, A., Post, A., Akay, Y. M., Jneid, H., Paniagua, D., Denktas, A., et al. (2016). Acoustic detection of coronary occlusions before and after stent placement using an electronic stethoscope. Entropy, 
18(8), 281.

23. Sharples, L., Hughes, V., Crean, A., Dyer, M., Buxton, M., Goldsmith, K., et al. (2007). Cost-effectiveness of functional cardiac testing in the diagnosis and management of coronary artery disease: a randomised controlled trial. The CECaT trial. HEALTH TECHNOLOGY ASSESSMENT-SOUTHAMPTON-, 11(49).

24. Thom, H., West, N. E., Hughes, V., Dyer, M., Buxton, M., Sharples, L. D., et al. (2014). Cost-effectiveness of initial stress cardiovascular MR, stress SPECT or stress echocardiography as a gate-keeper test, compared with upfront invasive coronary angiography in the investigation and management of patients with stable chest pain: mid-term outcomes from the CECaT randomised controlled trial. BMJ open, $4(2)$.

25. Runza, G., La Grutta, L., Alaimo, V., Evola, S., Re, F. L., Bartolotta, T., et al. (2007). Comprehensive cardiovascular ECG-gated MDCT as a standard diagnostic tool in patients with acute chest pain. 64(1), 41-47.

26. Sculpher, M., \& Drummond, M. J. E. e. i. h. c. M. t. w. p. (2001). The role and estimation of productivity costs in economic evaluation. 94-112.

27. Javan-Noughabi, J., Rezapour, A., Kassani, A., Hatam, N., \& Ahmadloo, N. (2018). The costeffectiveness of neoadjuvant chemotherapy in women with locally advanced breast cancer: Adriamycin and cyclophosphamide in comparison with paclitaxel and gemcitabine. Journal of research in medical sciences: the official journal of Isfahan University of Medical Sciences, 23, 57.

28. Hatam, N., Askarian, M., Javan-Noghabi, J., Ahmadloo, N., \& Mohammadianpanah, M. (2016). Costutility of "Doxorubicin and Cyclophosphamide" versus "Gemcitabine and Paclitaxel" for treatment of patients with breast cancer in Iran. Asian Pacific Journal of Cancer Prevention, 16(18), 8265-8270.

29. Darba, S., Safaei, N., Mahboub-Ahari, A., Nosratnejad, S., Alizadeh, G., Ameri, H., et al. (2020). Direct and Indirect Costs Associated with Coronary Artery (Heart) Disease in Tabriz, Iran. Risk Management and Healthcare Policy, 13, 969.

30. Lee, S.-P., Jang, E. J., Kim, Y.-J., Cha, M.-J., Park, S.-Y., Song, H. J., et al. (2015). Cost-effectiveness of coronary CT angiography in patients with chest pain: comparison with myocardial single photon emission tomography. Journal of Cardiovascular Computed Tomography, 9(5), 428-437.

31. Lee, D. S., Jang, M. J., Cheon, G. J., Chung, J.-K., \& Lee, M. C. (2002). Comparison of the costeffectiveness of stress myocardial SPECT and stress echocardiography in suspected coronary artery disease considering the prognostic value of false-negative results. Journal of nuclear cardiology, $9(5)$, 515-522.

32. Hlatky, M. A., Shilane, D., Hachamovitch, R., DiCarli, M. F., \& Investigators, S. (2014). Economic outcomes in the study of myocardial perfusion and coronary anatomy imaging roles in coronary artery disease registry: the SPARC study. Journal of the American College of Cardiology, 63(10), 1002-1008.

33. Van der Wall, E. (2014). Cost analysis favours SPECT over PET and CTA for evaluation of coronary artery disease: the SPARC study. Netherlands Heart Journal, 22(6), 257-258. 
34. Ferreira, A. M., Marques, H., Gonçalves, P. A., \& Cardim, N. (2014). Cost-effectiveness of different diagnostic strategies in suspected stable coronary artery disease in Portugal. Arquivos brasileiros de cardiologia, 102(4), 391-402.

35. Secretariat, M. A. (2010). Stress Echocardiography for the Diagnosis of Coronary Artery Disease: An Evidence-Based Analysis. Ontario Health Technology Assessment Series, 10(9), 1.

36. Sicari, R., Nihoyannopoulos, P., Evangelista, A., Kasprzak, J. D., Lancellotti, P., Poldermans, D., et al. (2009). Stress echocardiography expert consensus statement-executive summary: european association of echocardiography (a registrated branch of the ESC). European heart journal, 30(3), 278-289.

37. Stojanovic, I., Schneider, J. E., \& Cooper, J. (2019). Cost-impact of cardiac magnetic resonance imaging with Fast-SENC compared to SPECT in the diagnosis of coronary artery disease in the US. Journal of medical economics, 22(5), 430-438.

38. Forster, T., McNeill, A. J., Salustri, A., Reijs, A. E., El-Said, E.-S. M., Roelandt, J. R., et al. (1993). Simultaneous dobutamine stress echocardiography and technetium- $99 \mathrm{~m}$ isonitrile single-photon emission computed tomography in patients with suspected coronary artery disease. Journal of the American College of Cardiology, 21(7), 1591-1596.

39. Javan-Noughabi, J., Rezapour, A., Hajahmadi, M., \& Alipour, V. (2019). Cost-effectiveness of singlephoton emission computed tomography for diagnosis of coronary artery disease: A systematic review of the key drivers and quality of published literature. Clinical Epidemiology and Global Health, 7(3), 389-395.

40. Bedetti, G., Pasanisi, E. M., Pizzi, C., Turchetti, G., \& Loré, C. (2008). Economic analysis including longterm risks and costs of alternative diagnostic strategies to evaluate patients with chest pain.

Cardiovascular Ultrasound, 6(1), 21.

\section{Tables}

Table1 demographic characteristics of patients

\begin{tabular}{|c|c|c|c|}
\hline \multirow{2}{*}{\multicolumn{2}{|c|}{ Variation }} & \multicolumn{2}{|c|}{ Diagnostic modalities } \\
\hline & & \multirow{2}{*}{$\begin{array}{c}\text { SPECT } \\
60.84 \pm 56.11 \\
\end{array}$} & \multirow{2}{*}{$\begin{array}{c}\text { Stress echocardiography } \\
56.89 \pm 53.16 \\
\end{array}$} \\
\hline Age & & & \\
\hline \multirow[t]{2}{*}{ Sex } & Men & $\% 44$ & $\% 37$ \\
\hline & Women & $\% 56$ & $\% 63$ \\
\hline \multirow[t]{2}{*}{ Marital status } & Married & $\% 61$ & $\% 55$ \\
\hline & Single & $\% 39$ & $\% 45$ \\
\hline \multirow[t]{2}{*}{ Education } & University education & $\% 40$ & $\% 43$ \\
\hline & Non-university education & $\% 60$ & $\% 57$ \\
\hline \multicolumn{2}{|c|}{ Current smoker } & $\% 15$ & $\% 12$ \\
\hline \multicolumn{2}{|c|}{ Diabetes } & $\% 10$ & $\% 8$ \\
\hline \multicolumn{2}{|c|}{ Hypertension } & $\% 18$ & $\% 19$ \\
\hline \multirow[t]{3}{*}{ pretest likelihood of disease } & Low risk & $\% 61$ & $\% 67$ \\
\hline & Intermediate risk & $\% 37$ & $\% 32$ \\
\hline & High risk & $\% 2$ & $\% 1$ \\
\hline
\end{tabular}


Table 2 the cost items for CAD diagnosis

\begin{tabular}{|c|c|c|c|}
\hline \multicolumn{2}{|l|}{ Costs } & \multicolumn{2}{|c|}{ Diagnostic modalities (Average costs) } \\
\hline & & \multirow{2}{*}{$\begin{array}{l}\text { SPECT } \\
233.34\end{array}$} & \multirow{2}{*}{$\begin{array}{l}\text { Stress echocardiography } \\
131.8\end{array}$} \\
\hline Medical direct costs & diagnostic modalities & & \\
\hline & Visits & 9.1 & 9.3 \\
\hline & surgical costs & 233.75 & 201.8 \\
\hline & Consumables & 126.4 & 110 \\
\hline & Drugs & 9 & 11.6 \\
\hline & ECG & 6.4 & 6.7 \\
\hline & laboratory tests & 8.2 & 8.5 \\
\hline & Hoteling & 114.5 & 103.3 \\
\hline & Total & 740.69 & 583 \\
\hline \multirow[t]{4}{*}{ Non-medical direct costs } & Traveling & 49.7 & 43 \\
\hline & Accommodation & 19.8 & 18.6 \\
\hline & Phone & 30.1 & 22.9 \\
\hline & Total & 99.6 & 84.5 \\
\hline \multirow[t]{3}{*}{ Indirect costs } & For patients & 88.7 & 76.3 \\
\hline & $\begin{array}{l}\text { For patient's } \\
\text { accompany }\end{array}$ & 41.5 & 38 \\
\hline & Total & 130.2 & 114.3 \\
\hline
\end{tabular}

Table 3 The result of cost -effectiveness analysis of SPECT vs Stress echocardiography

\begin{tabular}{|r|r|r|r|r|r|}
\hline $\begin{array}{r}\text { Diagnostic } \\
\text { modalities }\end{array}$ & $\begin{array}{r}\text { Cost } \\
\text { (USD) }\end{array}$ & Effectiveness & $\Delta \boldsymbol{C}$ & $\Delta \boldsymbol{E}$ & $\begin{array}{l}\text { SPECT vs Stress } \\
\text { echocardiography }\end{array}$ \\
\hline $\begin{array}{r}\text { Stress } \\
\text { Echo }\end{array}$ & 316.3 & 0.84 & & & Dominated \\
\hline SPECT & 656.6 & 0.69 & 340.3 & -0.16 & \\
\hline
\end{tabular}

$\Delta \mathrm{C}=$ cost difference

$\Delta \boldsymbol{E}=$ effectiveness difference

\section{Figures}




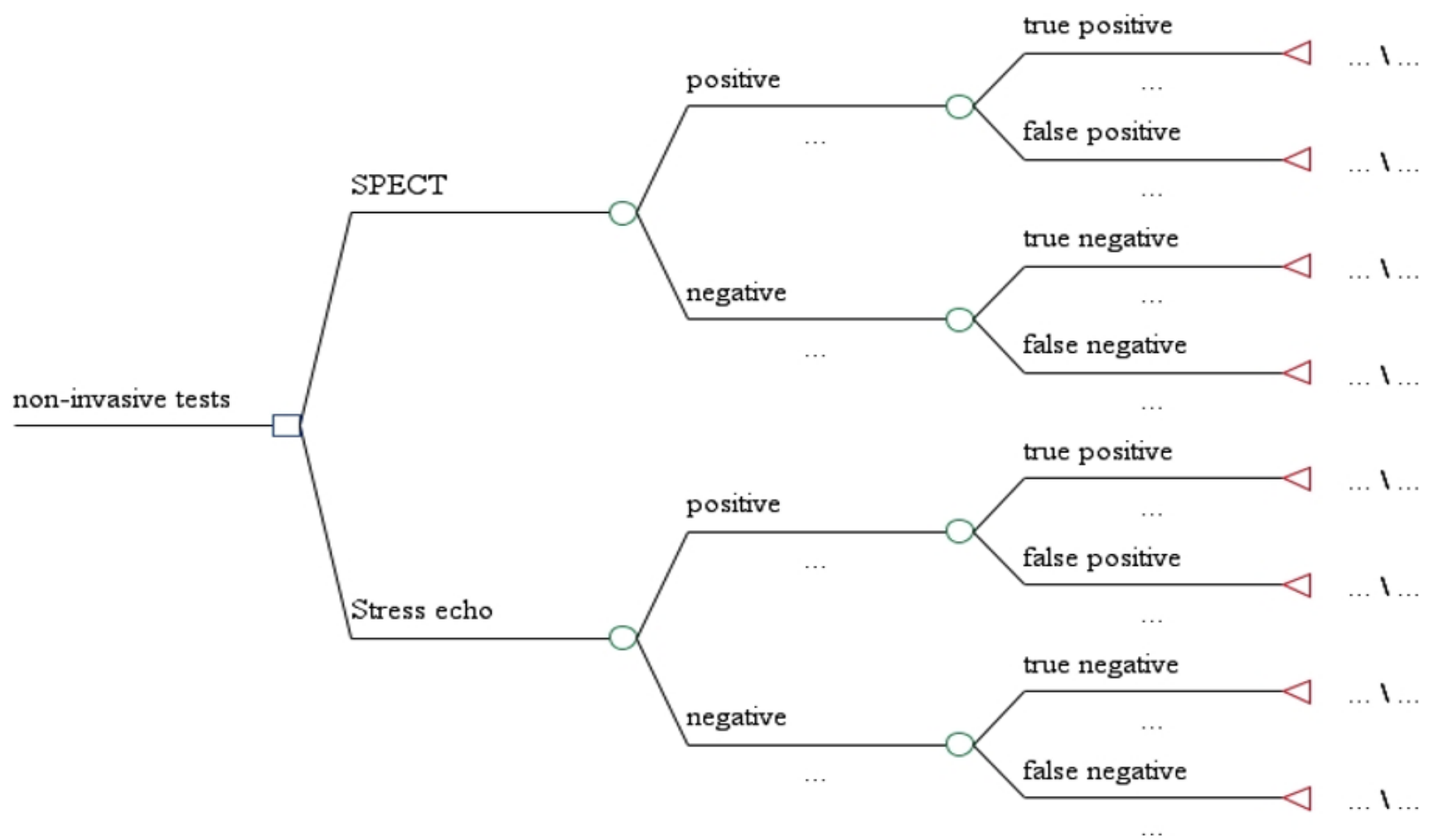

\section{Figure 1}

Decision tree algorithm for the cost-effectiveness of SPECT versus Stress echocardiography in diagnosis of CAD 


\section{Cost-Effectiveness Analysis}

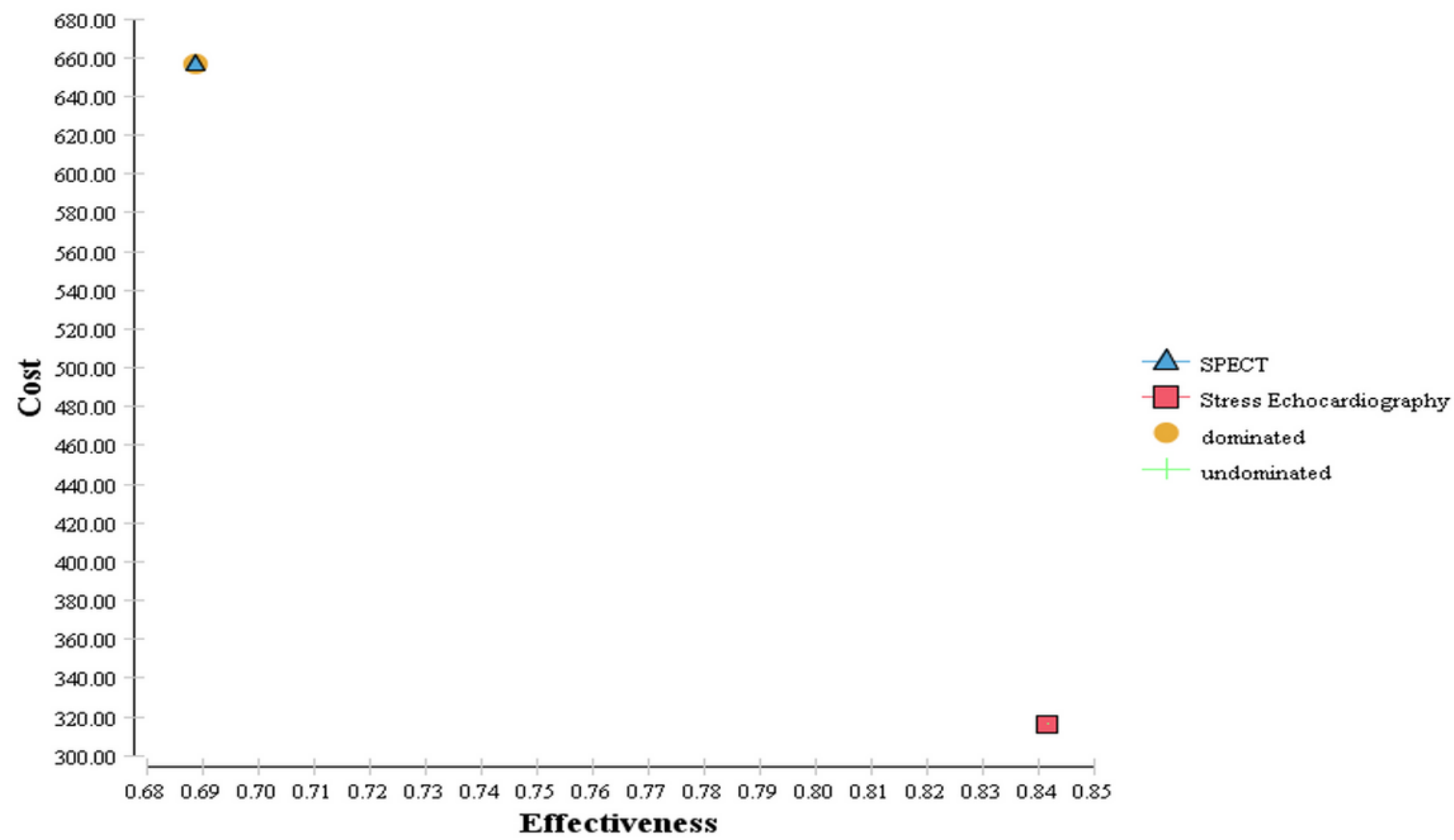

Figure 2

Cost-effectiveness analysis of SPECT versus Stress echocardiography in diagnosis of CAD 


\section{Tornado Analysis (ICER)}

True negative in branch of stress echo

True negative in branch of SPECT

True positive in branch of SPECT

Cost of True positive in branch of SPECT

True positive in branch of stress echo

Cost of true negative in branch of stress echo

Cost of True negative in branch of SPECT

Cost of false negative in branch of SPECT

Cost of true positive in branch of stress echo

Cost of false negative in branch of stress echo

Cost of false positive in branch of SPECT

Cost of false positive in branch of stress echo

EV: -22503056

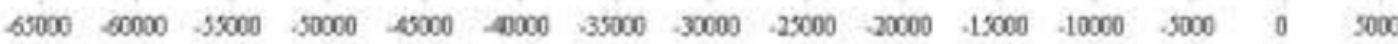

\section{Figure 3}

Tornado diagram for one-way sensitivity analysis 